\title{
Dynamical Local Lattice Instability Triggered High $T_{\mathrm{c}}$ Superconductivity
}

\author{
J. RANNINGER*
}

Institut Néel, CNRS and Université Joseph Fourier, BP 166, 38042 Grenoble cedex 9, France

High $T_{\mathrm{c}}$ cuprate superconductors are characterized by two robust features: their strong electronic correlations and their intrinsic dynamical local lattice instabilities. Focusing on exclusively that latter, we picture their parent state in form of a quantum vacuum representing an electronic magma in which bound diamagnetic spin-singlet pairs pop in and out of existence in a Fermi sea of itinerant electrons. The mechanism behind that resides in the structural incompatibility of two stereo-chemical configurations $\mathrm{Cu}^{\mathrm{II}} \mathrm{O}_{4}$ and $\mathrm{Cu}^{\mathrm{III}} \mathrm{O}_{4}$ which compose the $\mathrm{CuO}_{2}$ planes. It leads to spontaneously fluctuating $\mathrm{Cu}-\mathrm{O}-\mathrm{Cu}$ valence bonds which establish a local Feshbach resonance exchange coupling between bound and unbound electron pairs. The coupling, being the only free parameter in this scenario, the hole doping of the parent state is monitored by varying the total number of unpaired and paired electrons, in chemical equilibrium with each other. Upon lowering the temperature to below a certain $T^{*}$, bound and unbound electron pairs lock together in a local quantum superposition, generating transient localized bound electron pairs and a concomitant opening of a pseudo-gap in the single-particle density of states. At low temperature, this pseudo-gap state transits via a first order hole doping induced phase transition into a superconducting state in which the localized transient bound electron pairs get spatially phase correlated. The mechanism driving that transition is a phase separation between two phases having different relative densities of bound and unbound electron pairs, which is reminiscent of the physics of ${ }^{4} \mathrm{He}-{ }^{3} \mathrm{He}$ mixtures.

DOI: 10.12693 /APhysPolA.127.176

PACS: 74.72.-h, 74.20.Mn, 71.38.Mx

\section{Introduction}

Quite independent on any microscopic mechanism leading to superconductivity, this phenomenon is generated by establishing a macroscopic coherent quantum state in which an ensemble of transient bosonic charge carriers (composed of diamagnetic electron-pairs), having arbitrary phases in the parent state above $T_{\mathrm{c}}$, undergoes a global spontaneous symmetry breaking (SSB). The arbitrary phases of these virtual bosonic entities are thereby locked together into a unique global (though arbitrary) phase, the excitations of which are symmetry restoring collective Goldstone modes. In a current carrying state their existence assures the persistence of the resistanceless conduction through the Anderson-Higgs mechanism, by which they contribute to set up a longitudinal component of the electromagnetic vector potential driving this current, as recently reviewed [1] in commemorating the centennial anniversary of the discovery of superconductivity [2]. The value of the critical temperature $T_{\mathrm{c}}$ at which a super-flow sets in, depends however sensitively on how this SSB comes about in (i) forming finite amplitudes of individual bosonic entities and (ii) establishing the phase coherence between them in order to construct a macroscopic coherent quantum state. There are two ways for that to happen.

(I) When the strength of the inter-pair phase correlations, locking together the bosonic entities is large compared to the pairing energy. This is the case for BCS

\footnotetext{
*e-mail: julius.ranninger@neel.cnrs.fr
}

superconductors. The interaction between the electrons, monitored by the exchange of a phonon, is too weak to guarantee real space pairing. Yet, the ensemble of such virtual pairs, existing in form of transient Cooper pairs in momentum space, situated in a thin layer around the Fermi surface and having arbitrary phases, can be phase-locked into a macroscopic coherent quantum state through a collective process [3]. It provides the required strength for pairing, mediated by inter-pair phase correlations, engaging simultaneously a macroscopic number of transient Cooper pairs. Its resulting $T_{\mathrm{c}}$ is controlled by the zero temperature pairing amplitude $\Delta(0)$, tantamount to the energy of the single-particle gap $\Delta(0) \approx$ $1.76 k_{\mathrm{B}} T_{\mathrm{c}}^{\mathrm{BCS}}$ with $T_{\mathrm{c}}$ being given by

$$
\begin{aligned}
& T_{\mathrm{c}}^{\mathrm{BCS}} \approx \omega_{\mathrm{D}} \exp \left(-\frac{1}{\lambda /(1+\lambda)-\mu^{*}}\right), \\
& \mu^{*}=\mu /\left[1+\mu \ln \left(\varepsilon_{\mathrm{F}} / \omega_{\mathrm{D}}\right)\right], \\
& \mu-\lambda=\rho\left(\varepsilon_{\mathrm{F}}\right)\left\langle V_{\mathrm{el}-\mathrm{ph}}(q, \omega=0)\right\rangle_{\mathrm{FS}}, \\
& V_{\mathrm{el}-\mathrm{ph}}(q, \omega=0)=\frac{4 \pi e^{2}}{q^{2} \varepsilon(q, \omega=0)} .
\end{aligned}
$$

$T_{\mathrm{c}}$ sensitively depends on the difference between the attractive phonon-mediated electron-electron interaction $\lambda$ and the repulsive bare Coulomb interaction $\mu$, given by the electron lattice coupling $V_{\mathrm{el}-\mathrm{ph}}(q, \omega=0)$. Appearing in form of the average over the Fermi surface, $V_{\text {el-ph }}$ contributes predominantly through its small $q$-components of the static dielectric function $\varepsilon(q, \omega=0)$. For $\mathrm{q}=0$ having to be positive, in order to assure global crystalline stability, this renders $\mu-\lambda$ repulsive rather than attractive. Pairing in the BCS scenario finally occurs because, generating the superconducting state in such a collective 
process, it is the screened Coulomb interaction $\mu^{*}$ rather than the bare one which controls it [4] and for which $\mu^{*}-\lambda \leq 0$. But $\varepsilon(0,0)$ having to be positive still puts a stringent condition on obtaining sizeable values of $T_{\mathrm{c}}^{\mathrm{BCS}}$. Setting optimally $\mu=\lambda$ and varying the Debye frequency $\omega_{\mathrm{D}}$ in Eq. (1) in order to optimize the value of $T_{\mathrm{c}}^{\mathrm{BCS}}$, one obtains a maximal $T_{\mathrm{c}}^{\mathrm{BCS}}(\max )=\varepsilon_{\mathrm{F}} e^{-(4+3 / \lambda)}$, which for typical values of $\lambda$ and $\varepsilon_{\mathrm{F}}$ can barely exceed $30 \mathrm{~K}$.

Pines and Nozieres [5] pointed out that the causality of the response of the system's internal total charge to an external test charge, given by the dielectric function $\varepsilon(\boldsymbol{q}, \omega)$, requires that it is the inverse of it rather than the dielectric function itself which has to obey the KramersKronig relation. From that, Kirshnitz [6] concluded that, together with $\operatorname{Im} \varepsilon(\boldsymbol{q}, \omega=0) \leq 0$, a negative finite momentum $\varepsilon(\boldsymbol{q}, 0)$ not only is not incompatible with overall crystalline stability, but can in fact over-screen the repulsive Coulomb interaction by dynamical structural instabilities triggering diamagnetic pairing correlations on a local scale $[7,8]$. With this insight the systematic search for materials with incipient crystalline instabilities became a priority $[9,10]$. A15 compounds, showing displacive Martensitic lattice instabilities and attaining a $T_{\mathrm{c}}=25 \mathrm{~K}$ in $\mathrm{Nb}_{3} \mathrm{Ge}$ [11] re-enforced this strategy. Similar reasoning, in the early 1980ties, led our group in Grenoble to investigate transition metal oxides, such as $\mathrm{Ti}_{4} \mathrm{O}_{7}$ [12], which showed that their Fermi sea of bare itinerant electrons was unstable towards a charge disproportionated charge density wave (CDW) composed of alternating $\mathrm{Ti}(\mathrm{III})-\mathrm{Ti}(\mathrm{III})$ and $\mathrm{Ti}(\mathrm{IV})-\mathrm{Ti}(\mathrm{IV})$ diatomic molecular complexes housed inside deformable octahedral ligand environments. Given that the localized diamagnetic spin-singlet pairs on such molecular sites form small Bipolarons, we proposed that upon doping $\mathrm{Ti}_{4} \mathrm{O}_{7}$ with $\mathrm{V}$ or Sc the insulating CDW state could be destabilized and make the system transit into a bipolaronic superconductor [13], assuming that such a priori localized bipolarons could be rendered itinerant and result in a super-fluid phase with a $T_{\mathrm{c}}$ determined by their mass density.

(II) When the strength of the inter-pair phase correlations is small compared to the pairing energy. In that case bound real-space pairs are formed without having to invoke their condensation, such as in potential bipolaronic superconductors. In order for those bound electron pairs to condense into a super-fluid state, analogous to that of super-fluid ${ }^{4} \mathrm{He}(\mathrm{II})$ with a $T_{\mathrm{c}}$ being controlled by the mass density of super-fluid charge carriers, these bosonic entities have: (i) to be locally well defined individual particles, not or only weakly overlapping with each other and (ii) to exist in form of itinerant states. On a very general level, the scheme of real space pair superconductivity had been addressed on the basis of the negative $U$ Hubbard model by numerous authors as was reviewed [14] in the early years of the high temperature superconductivity (HTSC) era, when it was used to account for the crossover from a BCS state to a Bose-Einstein condensate (BEC) in the so-called phase fluctuation scenario [15]. Given that in real materials the pairing of electrons is generally generated by strong local lattice deformations which trap electrons into small localized bipolarons, it became evident that such local pairs could not exist in form of itinerant charge carriers [16]. Searching a way out of this dilemma led me to propose that resonating localized bipolarons could achieve a phase fluctuation driven superconducting state on the basis of their transient nature. It is that which permits them through their amplitude fluctuations to lock together their respective phases in a macroscopic coherent quantum state. The intrinsic metastability of the cuprate HTSCs, discovered in 1986 [17], provides us with this prerequisite encountering transient localized bound electron-pairs well above $T_{\mathrm{c}}$.

Physical realizations of BEC driven superconductivity had been known for some time to occur in diamagnetic insulating, respectively semiconducting, parent compounds upon substitutionally doping them with cations, or rendering them sub-stoichiometric. Their cation-ligand configurations are capable to sustain superconducting diamagnetic pairing fluctuations in very dilute concentrations of charge carriers, typically around $10^{20}$ per $\mathrm{cm}^{3}$ in compounds such as $\mathrm{SrTiO}_{3-x}$ with a $T_{\mathrm{c}} \approx 0.3 \mathrm{~K}[18]$. Substitutionally doped $\mathrm{SrTiO}_{3}$ i.e. $\mathrm{SrTi}_{0.97} \mathrm{Zr}_{0.03} \mathrm{O}_{3}$ [19], presents a superconducting $\mathrm{BEC}$ with $T_{\mathrm{c}} \approx 0.07 \mathrm{~K}$ for a charge carrier concentration as low as of $4 \times 10^{15}$ per $\mathrm{cm}^{3}$. Eagles [20] pointed out that this result is compatible with the classical BCS pair exchange mechanism in the limit of very low carrier concentrations. Leggett [21] a few years later, in a quest to describe super-fluidity of ${ }^{3} \mathrm{He}$, showed how the BCS ground state wave-function in the weak pairing regime describes in the limit of very low carrier concentrations a BEC of real space pairs. Achieving in that scenario higher concentrations with well defined local bosonic bound electron pairs of not too heavy masses and thus high $T_{\mathrm{c}}$ 's however had been hampered for many years.

\section{Resonating bipolarons}

Cuprate HTSCs manage to evade the problem related to the mobility of real space pairs in crystalline materials and a $T_{\mathrm{c}}$ controlled by their mass density. Their non-Fermi liquid electronic magma parent state at high temperatures can be monitored over a large regime of carrier concentrations upon changing, in the chemical synthesis, the relative composition of the basic ingredients: the stereo-chemical $\mathrm{Cu}(\mathrm{II}) \mathrm{O}_{4}$ and $\mathrm{Cu}(\mathrm{III}) \mathrm{O}_{4}$ molecular clusters.

There are two energy scales which control the interdependence of (i) the onset of the pseudo-gap state out of a high temperature electronic magma parent state and (ii) the superconducting state, evolving out of this pseudo-gap state at low temperatures through a holedoping induced phase first order phase transition.

The first one is given by the pairing energy, characterized by the temperature $T^{*}$ signalling the opening 
of pseudo-gap in the single-particle density of states. It is related to the generation of transient localized diamagnetic spin-singlet pairs on competing with each other $\mathrm{Cu}(\mathrm{II})-\mathrm{O}-\mathrm{Cu}(\mathrm{II})$ and $\mathrm{Cu}(\mathrm{III})-\mathrm{O}-\mathrm{Cu}(\mathrm{III})$ valence bonds, generating a finite fluctuating pairing amplitude, which monotonously decreases with increasing hole doping.

The second one is given by the energy, required to phase lock together these transient localized electron pairs on adjacent fluctuating molecular clusters. This is achieved by their faculty to spontaneously decay into a pair of itinerant electrons, which establishes this phase correlation through the Andreev type scattering processes.

The strongly hole doping dependent competition between inter-pair and intra-pair phase correlations dictates the phase transition by which the superconducting state, composed of spatially phase correlated transient localized bound electron-pairs, transits into the insulating pseudo-gap state with phase uncorrelated such bound electron-pairs.

When the pairing energy outweighs the energy related to the phase stiffness, which links adjacent transient pairs as is the case for low doped cuprate HTSCs up to the optimally doped ones, the decreasing with hole doping $x$ pairing energy $k_{\mathrm{B}} T^{*}(x)$ weakens the local intra-pair phase rigidity and thereby permits to strengthen the inter-pair phase stiffness. As a consequence, $T_{\mathrm{c}}(x)$ increases with increasing $x$. Being controlled exclusively by phase fluctuations, $T_{\mathrm{c}}$ scales with the mass density of the transient bound electron pairs, as experimentally established in Uemura's universal plot of $T_{\mathrm{c}}$ versus $n_{\mathrm{s}} / m_{\mathrm{s}}$ [22], obtained from positron annihilation studies. Upon approaching the optimal doping $x_{\mathrm{opt}}$, a maximal value of $T_{\mathrm{c}}\left(x_{\mathrm{opt}}\right)$ is reached when amplitude and phase fluctuations corroborate optimally to construct a state in which both of those fluctuations are simultaneously minimized. Beyond $x_{\mathrm{opt}}$, the pairing energy $k_{\mathrm{B}} T^{*}(x)$, decreasing with increasing hole doping, takes over the control of a BCS like superconducting state triggered by amplitude fluctuations.

These features are to a certain extent compatible with both of the two robust characteristics of the cuprate HTSCs: (i) their intrinsic dynamical local instabilities of the crystalline lattice and (ii) their strong electronic correlations which characterise a hole doped Mott insulator, resulting in Anderson's resonating valence bond (RVB) scenario [23]. Both of these scenarios lead one to a picture in which bare itinerant electrons, moving in planar $\mathrm{CuO}_{2}$ structures, get momentarily bound in form of resonating diamagnetic spin-singlet pairs on plaquettes composed of four $\mathrm{Cu}$ cations. Under certain provisos, the RVB scenario can be mapped into the phenomenological effective boson-fermion model (BFM) [24] which had been advocated prior to the discovery of the cuprate HTSCs to capture the physics of metastability driven superconductivity. In an early mean field analysis [25] of the BFM it indicated for the first time the potentiality of a pseudo-gap state controlled by amplitude fluctuations in a system of intrinsically localized transient bipolarons.
The full implications of that had however been recognized [26] only once the cuprates superconductors had been discovered.

Our investigations during the past two decades of meta-stability driven pairing in the cuprates on the basis of the BFM permitted us to predict the salient features of this pseudo-gap state: (1) the anomalous temperature dependence of this pseudo-gap [27], (2) the remnant Bogoliubov modes [28] and (3) to account for its transient Meissner effect [29]. With this background we restrict ourselves now to focus on the scenario of lattice metastability triggered superconductivity, which requires, to start with, to conjecture a corresponding to it quantum vacuum parent state. The physics of the cuprate HTSCs, manifest in their experimentally established temperature-doping dependent phase diagram, then has to be derivable from such a parent state on the basis of very general symmetry breaking processes.

The quantum vacuum parent state of the cuprate HTSCs is generated by chemical synthesis at high temperatures. It describes a solid solution in an out of thermal equilibrium high entropy state, which arises from incompatible $\mathrm{CuO}_{4}$ square planar stereo-chemical complexes $\mathrm{Cu}(\mathrm{II}) \mathrm{O}_{4}$ with a $\mathrm{Cu}-\mathrm{O}$ bond-length of $1.93 \AA$, and $\mathrm{Cu}(\mathrm{III}) \mathrm{O}_{4}$ with a $\mathrm{Cu}-\mathrm{O}$ bond-length of $1.83 \AA$. Both of them are constrained to coexist in the $\mathrm{CuO}_{2}$ layers, sandwiched between the layered charge reservoirs. Chemical reactivity between them, controlled by the covalency of their $\mathrm{Cu}-\mathrm{O}-\mathrm{Cu}$ bonds, stabilises the overall crystal structure kinetically by quantum fluctuations of the $\mathrm{Cu}-\mathrm{O}$ bond-length, oscillating between 1.93 and $1.83 \AA$. This is evidenced experimentally in the double peak structured PDF (pair-distribution function) [30] and in the splitting of the $\mathrm{Cu}-\mathrm{O}-\mathrm{Cu}$ bond stretch mode [31]. The fluctuations of the bond-length go hand in hand with double charge fluctuations on such deformable plaquettes favouring textured meso-structures, as seen in STM-IS (scanning tunnelling microscope imaging spectroscopy) [3234] (see Fig. 1). The spectral distribution of the binding energy of the bosonic bound pairs on those textured plaquettes shows an isotope shift in $\mathrm{d}^{2} / \mathrm{d} V^{2}$-imaging studies, upon replacement of $\mathrm{O}^{16}$ by $\mathrm{O}^{18}$ [35]. This, together with the strong positive isotope effect of the pairing energy $k_{\mathrm{B}} T^{*}(x)$ [36] and its being correlated to the pressure induced $\mathrm{Cu}-\mathrm{O}$ bond stretch mode frequency [37] are strong indications that pairing in the cuprate HTSCs derive primarily from their intrinsic crystalline metastability.

The local dynamical lattice deformations, involving directionally oriented fluctuating $\mathrm{Cu}-\mathrm{O}-\mathrm{Cu}$ bonds, are randomly oriented along the $a$ and $b$ direction. This breaks the rotational as well as translational local lattice symmetry on an atomic length scale, and thereby evades any onset of long range translational symmetry breaking, which could hinder the stabilization of a phase correlated superfluid state.

Given these experimentally established features of spontaneous dynamical local lattice instabilities in the 
cuprate HTSCs, we visualize their high temperature quantum vacuum parent state as one in which bound spin-singlet pairs pop in and out of existence in an underlying Fermi sea of itinerant electrons. As we shall see below, the electrons thereby get absorbed in the construction of localized bosonic pairs with well defined pairing amplitudes fluctuating around a non-zero amplitude below $T^{*}$. It is generated by a local symmetry breaking in which bound and unbound electron-pairs of that quantum vacuum engage in a locally phase locked quantum superposition.

The electrons having been eaten up in the construction of transient electron pairs display purely collective phase fluctuation Goldstone modes, which are controlled by the systems aspiration to condense into a macroscopic coherent quantum state, driven by a mechanism which could be ascribed to "quantum protection" [38, 39].

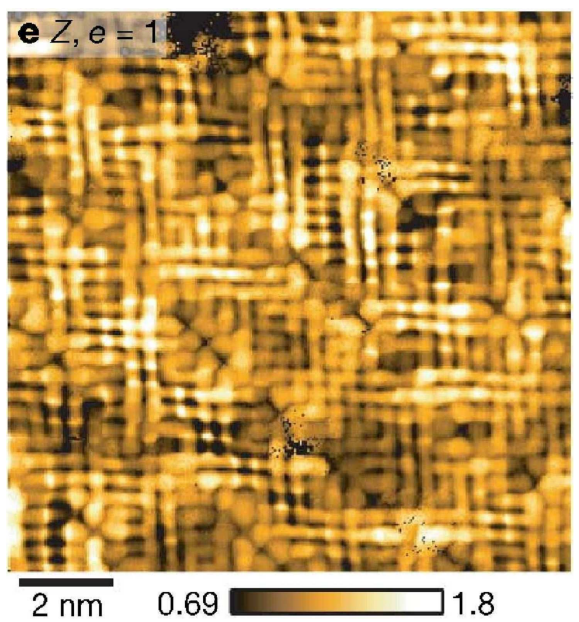

Fig. 1. Chequerboard segregation of holes (after Ref. [33]), evidenced in STM imaging studies.

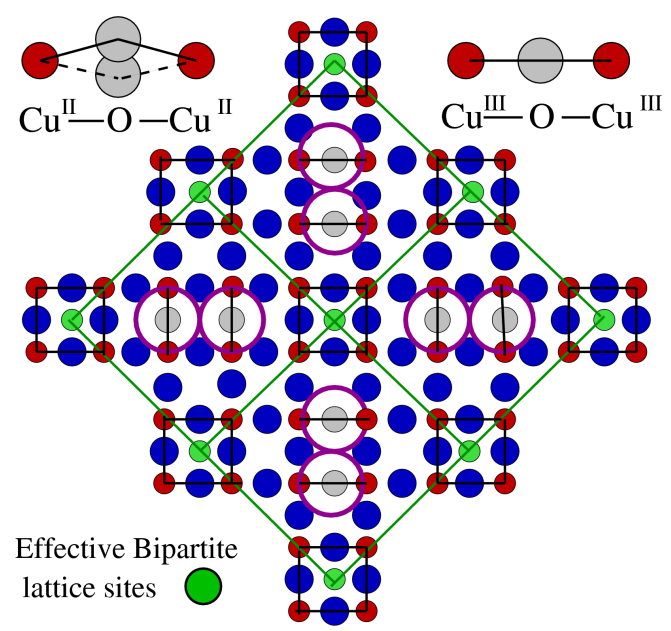

Fig. 2. An idealized bipartite lattice structure of the texturing of the charge distribution of the $\mathrm{CuO}_{2}$ planes. Itinerant electrons move on the sublattice which links effective lattice sites, given by the small filled green circles.
In order to formulate the scenario of metastability driven pairing of the HTSCs, let us picture the spontaneously induced texturing of the $\mathrm{CuO}_{2}$ layers on the basis of an idealized regular chequer-board structure bipartite lattice (see Fig. 2), composed of two inter-penetrating sub-lattices. Itinerant electrons, experiencing the locally fluctuating covalent $\mathrm{Cu}-\mathrm{O}-\mathrm{Cu}$ bonds in the $\mathrm{CuO}_{2}$ layers, get momentarily self-trapped in form of resonating Pauling covalent bonds [40], on dynamically fluctuating molecular clusters - square plaquettes composed of pairs of $\mathrm{Cu}-\mathrm{O}-\mathrm{Cu}$ bonds which fluctuate between $\mathrm{Cu}(\mathrm{III})-\mathrm{O}-$ $\mathrm{Cu}(\mathrm{III})$ and $\mathrm{Cu}(\mathrm{II})-\mathrm{O}-\mathrm{Cu}(\mathrm{II})$ stereo-chemical configurations. We know from the theory of many body small polaron physics that a homogeneous state for such a situation is unstable against texturing, driven by local quantum superposition of bound polarons (bipolarons) and quasi-free electron pairs on small clusters, composed of diatomic molecules [41]. In the cuprates this manifests itself in form of plaquettes composed of locally fluctuating $\mathrm{Cu}-\mathrm{O}-\mathrm{Cu}$ bonds, forming a sub-lattice " $\mathrm{A}$ ", which is embedded in a sub-lattice "B". The effective sites on that latter are given by plaquettes composed of rigid $\mathrm{Cu}(\mathrm{II})-\mathrm{O}-\mathrm{Cu}(\mathrm{II})$ bonds along which we envisage the electrons to move as quasi free itinerant particles. In the absence of any such texturing the electrons would form a half filled band metal for undoped cuprates (we neglect here any Hubbard on-site repulsion on the $\mathrm{Cu}$ ions). But in textured structures the freely moving electrons on sub-lattice "B", get momentarily self-trapped when they hop onto the dynamically deformable plaquettes on sub-lattice "A". When considering the dynamically undeformable plaquettes, forming the effective lattice sites of the sublattice together with the deformable cluster on sublattice "A", which they surround, two electrons on such effective sites of such a rescaled lattice structure exist simultaneously as bound and un-bound pairs. Driven by the intrinsic local molecular fluctuations, the exchange between the two is described by a Feshbach pair resonance coupling [42]: $g \sum_{i}\left(\rho_{i}^{+} \tau_{i}^{-}+\rho_{i}^{-} \tau_{i}^{+}\right)$. It accounts for the transfer of unbound electron pairs $\tau_{i}^{+}=c_{i \uparrow}^{\dagger} c_{i \downarrow}^{\dagger}$ from the metallic substructure on sub-lattice "B" to the insulating substructure on sub-lattice "A", on which these same electrons get momentarily bound into localized hard-core bosons in form of localized bound spin-singlet pairs $\rho_{i}^{+}$(represented by a pseudo-spin $\frac{1}{2}$ operators $\left.\left[\rho_{i}^{+}, \rho_{i}^{-}, \rho_{i}^{z}=\rho_{i}^{+} \rho_{i}^{-}-1 / 2\right]\right)$. And vice versa. It is in this dynamical process of transient pairing, induced by spontaneous local lattice instabilities that a charge deficiency occurring on the plaquettes of sub-lattice A, evidences in the STM-IS [33] the transiently bound hole pairs.

The interplay between the electrons in itinerant singleparticle states and in localized two-particle bound states, has been cast into an effective phenomenological bosonfermion model

$$
H_{\mathrm{BFM}}=\left(\varepsilon_{0}-\mu\right) \sum_{i \sigma} c_{i \sigma}^{\dagger} c_{i \sigma}+\left(\Delta_{\mathrm{B}}-2 \mu\right) \sum_{i}\left(\rho_{i}^{z}+\frac{1}{2}\right)
$$




$$
-\sum_{i \neq j \sigma} t\left(c_{i \sigma}^{\dagger} c_{j \sigma}+\text { H.c. }\right)+g \sum_{i}\left(\rho_{i}^{+} \tau_{i}^{-}+\rho_{i}^{-} \tau_{i}^{+}\right)
$$

in the early 1980ties in an attempt to obtain a super-fluid state of intrinsically localized small bipolarons. The idea behind that proposition was to work with resonating bipolarons which, albeit being localized quantities, could exist with locally fluctuating pairing amplitudes - generated by a crystalline metastability. Such a scenario introduces a dichotomy of the charge carriers, existing as both: free fermionic particles with an on-site energy $\varepsilon_{0}$ and bound bosonic pairs of them with an energy $\Delta_{\mathrm{B}}$. The two manifestations of the charge carriers, coexisting in chemical equilibrium with each other, require that the total number of the spin-singlet hard-core bosons and of the fermionic itinerant electrons, $n_{\text {tot }}=n_{\mathrm{F} \uparrow}+n_{\mathrm{F} \downarrow}+2 n_{\mathrm{B}}$, is conserved. It implies a chemical potential $\mu$, common to both subsystems. $n_{\mathrm{B}}, n_{\mathrm{F} \uparrow, \downarrow}$ denote the occupation numbers of the hard core-bosons and of the electrons with up and down spins. The strength of the Feshbach resonant exchange coupling $g / t$, in units of the electron hopping integral, is the only free parameter in this scenario. The effect of doping is controlled by the chemical potential which predominantly acts on the average density of bosonic charge carriers. For the cuprate HTSCs, in which the bare conduction electrons form a half-filled band in the undoped regime (we neglect any Hubbard onsite repulsion), we put $\varepsilon_{0}=\Delta_{\mathrm{B}}=0$. This implies that the exchange coupling between unbound and bound electron pairs occurs at the Fermi level of the bare itinerant electrons and results in $n_{\mathrm{F}}=n_{\mathrm{B}}=1$ when $\mu=0$.

\section{The anti-correlated doping dependence of $T^{*}(x)$ and $T_{\mathrm{c}}(x)$}

The salient features of high temperature superconductivity in this crystalline metastability driven scenario nucleate in the atomic limit of this model [43] and are described by the local physics of the parent state. Its Hilbert space consists of eight eigenvectors, made out of four fermionic states $|2\rangle,|3\rangle,|6\rangle,|7\rangle$ and four bosonic states, $|1\rangle,|4\rangle,|5\rangle,|8\rangle$, given by

$$
\begin{aligned}
& |2\rangle=\left|c_{\uparrow}^{\dagger}\right\rangle, \quad|3\rangle=\left|c_{\downarrow}^{\dagger}\right\rangle, \\
& |6\rangle=\left|c_{\uparrow}^{\dagger} \rho^{+}\right\rangle, \\
& |7\rangle=\left|c_{\downarrow}^{\dagger} \rho^{+}\right\rangle, \\
& |1\rangle=|0\rangle \\
& |4\rangle=(1 / \sqrt{2})\left[\mathrm{e}^{+\mathrm{i} \frac{\phi}{2}}\left|c_{\uparrow}^{\dagger} c_{\downarrow}^{\dagger}\right\rangle-\mathrm{e}^{-\mathrm{i} \frac{\phi}{2}}\left|\rho^{+}\right\rangle\right], \quad E_{4}=-g \\
& |5\rangle=(1 / \sqrt{2})\left[\mathrm{e}^{+\mathrm{i} \frac{\phi}{2}}\left|c_{\uparrow}^{\dagger} c_{\downarrow}^{\dagger}\right\rangle+\mathrm{e}^{-\mathrm{i} \frac{\phi}{2}}\left|\rho^{+}\right\rangle\right], \quad E_{5}=+g, \\
& |8\rangle=\left|c_{\uparrow}^{\dagger} c_{\downarrow}^{\dagger} \rho^{+}\right\rangle \text {, } \\
& E_{8}=0 \text {, }
\end{aligned}
$$

with corresponding eigen-energies $E_{i}$ and taking $\Delta_{\mathrm{B}}=$ $\varepsilon_{0}=0$.

The effect of the crystalline metastability on the electronic structure is controlled by an intra-pair phase rigidity, which locks together in a quantum superposition the phases of the two-particle states $|4\rangle$ and $|5\rangle$, describing: (i) bound electron-pairs momentarily occupying dynamically fluctuating molecular clusters in form of self-trapped bipolarons (favouring an insulating state) and (ii) unbound pairs of delocalized electrons passing momentarily through such fluctuating molecular clusters (favouring a metallic state). At temperatures above a certain $T^{*} \approx g$, the thermal fluctuations of the molecular clusters destroy this phase locking. Itinerant electrons then are scattered off from localized bosonic bound pairs, which spontaneously appear and disappear on such effective lattice sites and thereby loose any Fermi liquid properties. Decreasing the temperature to below $T^{*}$, the intra-pair phase locking (evidenced in the rapid increase of the correlation function $\left.\left\langle c_{\downarrow} c_{\uparrow} \rho^{+}\right\rangle=(1 / 2) \tanh (\beta g / 2)\right)$, linking localized bound pairs and unbound pairs on such individual fluctuating molecular clusters generates the polarizibility of such metastable system, which manifests itself in form:

(i) of an increasing number of electrons participating in transient pairing, $n_{p}=\left\langle c_{i \uparrow}^{\dagger} c_{i \downarrow}^{\dagger} c_{i \downarrow} c_{i \uparrow}\right\rangle=(1+\cosh (\beta g) /(6+$ $2 \cosh (\beta g)$ which in the zero temperature limit tends to $n_{p}=1 / 2$, i.e., twice its free particle value $n_{p}=1 / 4$ above $T^{*}$,

(ii) of the local pair susceptibility in the frequency-zero $\operatorname{limit} \chi\left(\mathrm{i} \omega_{n} \rightarrow 0\right)=\int_{0}^{\beta} \mathrm{d} \tau\left\langle T c_{\downarrow}(\tau) c_{\uparrow}(\tau) c_{\uparrow}^{\dagger}(0) c_{\downarrow}^{\dagger}(0) \mid\right\rangle=$ $\frac{1}{g}[\sinh (\beta g) / 3+\cosh (\beta g)]$, which approaches $1 / g$ when $T$ drops to below $T^{*}$.

This feature illustrates how the incipient polarizability of the parent state above $T^{*}$ generates, through dynamical symmetry breaking, finite amplitude pairs in an ensemble of competing stereo-chemical configurations, once forced by chemical synthesis, to form a regular crystal structure in which the electrons exist simultaneously as itinerant and as trapped into pair states. As a manifestation of that, the spectral properties of the single-particle excitations given by the local Green's function [43] exhibits a characteristic three-pole structure:

$$
\begin{gathered}
G_{\text {at }}\left(\mathrm{i} \omega_{n}\right)=-\int_{0}^{\beta} \mathrm{d} \tau \exp ^{\mathrm{i} \omega_{\mathrm{n}} \tau}\left\langle\mathrm{T}\left[\mathrm{c}_{\sigma}(\tau) \mathrm{c}_{\sigma}^{\dagger}(0)\right]\right\rangle= \\
{\left[\mathrm{i} \omega_{n}-\Sigma_{a t}\left(\mathrm{i} \omega_{n}\right)\right]^{-1}=\frac{Z^{\mathrm{F}}}{\mathrm{i} \omega_{n}}+\frac{\left[1-Z^{\mathrm{F}}\right] \mathrm{i} \omega_{n}}{\left[\mathrm{i} \omega_{n}\right]^{2}-g^{2}},} \\
\Sigma_{\text {at }}\left(\mathrm{i} \omega_{n}\right)=\frac{(1-Z) g^{2} \mathrm{i} \omega_{n}}{\left(\left[\mathrm{i} \omega_{n}\right]^{2}-Z g^{2}\right.} .
\end{gathered}
$$

The first term in $G_{\text {at }}\left(\mathrm{i} \omega_{n}\right)$, Eq. (7), derives from unbound electrons momentarily occupying such local dynamically fluctuating molecular sites with an energy equal to $\varepsilon_{0}=0$ and having a spectral weight $Z^{\mathrm{F}}=$ $2 /(3+\cosh \beta g)$. The second term in $G_{\text {at }}\left(i \omega_{n}\right)$ derives from locally bound electron pairs in bonding and antibonding states $|4\rangle$ and $|5\rangle$. These two contributions have the structure of the BCS spectral function, with the BCS gap being replaced by the exchange coupling $g$. From the behaviour of $Z^{\mathrm{F}}$ we notice that the almost temperature independent spectral weight of the central peak at frequency zero above $T^{*}$ abruptly decreases upon going to below $T^{*}$. Correlated to that, the spectral weight $\left(1-Z^{\mathrm{F}}\right)$ of the bonding and anti-bonding contribution abruptly increases. This feature of the local Green function contains the key to the mechanism resulting 
in the opening the pseudo-gap at $T^{*}$ in the single-particle density of states and to the anomalous temperature behaviour of the transport coefficients in the high temperature regime above $T^{*}$. In order to access these properties one has to incorporate this local physics of such fluctuating molecular clusters into an ensemble of such clusters in which the bare itinerant electrons move. Given the local nature of the Feshbach exchange coupling, a reliable approach to that has proven to be a dynamical mean field theory (DMFT) analyses [44]. We illustrate in Fig. 3 the evolution with temperature of the spectral function of the electrons at the wave-vector $\varepsilon=0$, which corresponds to the Fermi wave-vector before the Fermi surface was destroyed.

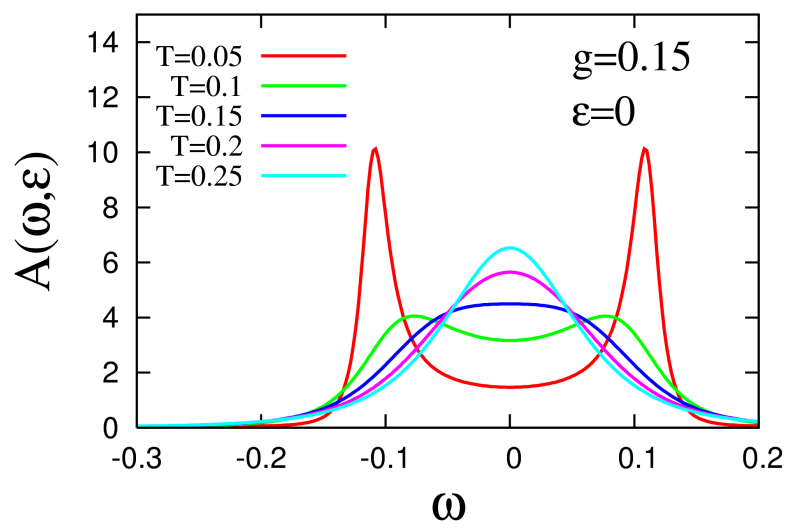

Fig. 3. Evolution with temperature of the spectral function for electrons at the hidden Fermi surface (after Ref. [44]) (characterized by an energy $\varepsilon=0$ ) for $g / t=0.15$. The curve with the highest maximum at $\omega=0$ corresponds to $T=0.5$.

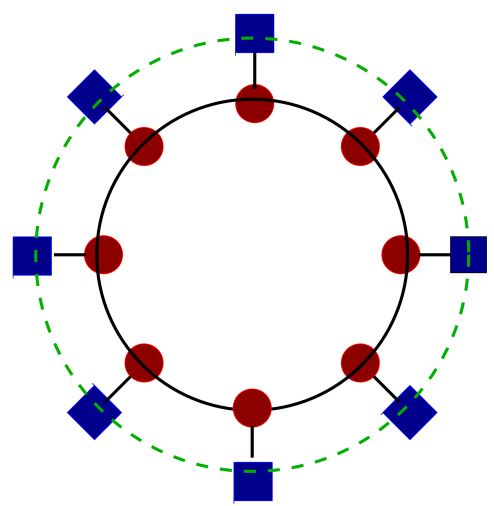

Fig. 4. A 1D exemplification of the BFM scenario on a closed 8-site ring with composite effective lattice sites. Itinerant electrons sitting on the circular sites in red on sublattice B move on the ring via inter-site hopping and also hop in pairs onto the pairing centres given by the square sites in blue on sublattice $\mathrm{B}$, where they get momentarily trapped in form of localized hard-core bosonic bound pairs.

The salient feature of this metastability driven pairing and its resulting from that low temperature superconducting, respectively insulating, state derives from the competition between (i) the local intra-pair phase correlations, which link the bound and unbound pairs on a given site (as described by the atomic limit of $H_{\mathrm{BFM}}$ ) and (ii) the inter-pair phase correlation, generated by the electron hopping which links the phases of the bound electron pair components of these transient local composite bosonic entities on neighbouring sites. In order to illustrate that, let us consider this problem on hand of a closed ring like structure, which presents both of the sublattices in terms of effective composite sites, such as shown in Fig. 4. Solving this BFM by an exact diagonalization study for such a ring like structure [45], we show on the left part of Fig. 5 the variation of $T^{*}$ and $T_{\phi}$, which characterizes the onset of local pairing (determining the onset of the pseudo-gap state) and of spatial phase correlations (determining the onset of superconductivity) for a fixed $g / t=0.5$ as a function of hole doping: $n_{\mathrm{B}}=4 / 8=0.5$ presenting the case of undoped cuprates and $n_{\mathrm{B}}$ between $3 / 8$ and 0 for the hole doped ones. The hole doping $x=0.5-n_{\mathrm{B}}$ is tracked by fixing the average number of bosonic bound pairs $n_{\mathrm{B}}$ on this ring. The hole doping appearing symmetric with respect to electron doping with $0.5 \leq n_{\mathrm{B}} \leq 1$ in this illustration of $T^{*}$ and $T_{\phi}$, results from having assumed that the pair exchange coupling between bound and unbound hole pairs is identical to that of bound and unbound electron pairs. This is evidently not so, since for electron doped systems the two stereo-chemical configurations which compete with each other are $\mathrm{Cu}(\mathrm{II}) \mathrm{O}_{4}$ and $\mathrm{Cu}(\mathrm{I}) \mathrm{O}_{4}$, the former having a square planar and the latter linear dumbbell stereo-chemical configurations. For the sake of the present illustration, where we concentrate on the robust qualitative features of the cuprates, we shall ignore this quantitative effect on the particle-hole asymmetry of the Feshbach exchange coupling.

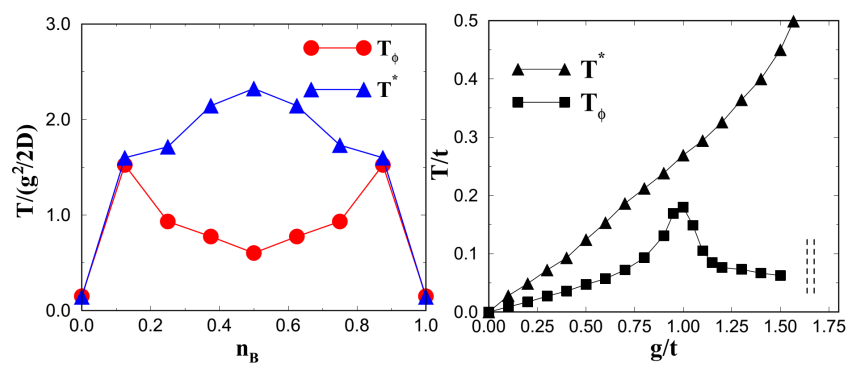

Fig. 5. $T^{*}$ and $T_{\phi}$ for a closed 8 site ring (after Ref. [45]) shown in Fig. 4 with composite effective lattice sites. The left part shows the doping dependence of $T^{*}$ and $T_{\phi}$ as a function of the average site occupation by bound pairs for $g / t=0.5$. The right part shows the variation of $T^{*}$ and $T_{\phi}$ for $n_{\mathrm{B}}=0.5$ as a function of $g / t$, which indicates the transition of the superconducting phase into the Bose phase glass insulator, when $g / t$ increase beyond unity.

$T^{*}$ in this finite size features of the BFM is determined by an abrupt decrease of the local intra-pair correlations given by $\left\langle\left|\rho_{i}^{+} \tau_{i}^{-}\right|\right\rangle$which shows upon decreasing $n_{\mathrm{B}}$ from 0.5 to 0 (increasing the hole doping 
from the underdoped to the overdoped systems) a steady monotonously decreasing behaviour. $T_{\phi}$ is determined by an equally abrupt increase of the long range phase coherence of the hard core bosonic bound pairs, given by $\left\langle\left|\rho_{\boldsymbol{q}}^{+} \rho_{\boldsymbol{q}}^{-}\right|\right\rangle$for $q=0$.

In Fig. 5, left part, we illustrate how upon decreasing $n_{\mathrm{B}}$ from 0.5 to $1 / 8$ (increasing the hole doping from zero up to the optimal doping rate) $T_{\phi}$ increases monotonously until it hits the monotoneously decreas$\operatorname{ing} T^{*}$ around $n_{\mathrm{B}}=1 / 8$. From there on, upon further increasing the hole doping (decreasing $n_{\mathrm{B}}$ ), $T_{\phi}$ becomes delimited by the pairing energy $k_{\mathrm{B}} T^{*}$, which displays features which are characteristic for BCS superconductors. The minimum of $T_{\phi}$ at $n_{\mathrm{B}}=0.5$ observed in this finite size system hints the system's tendency to transit into an insulating Bose glass state composed of spatially phase uncorrelated localized transient electron-pairs. For larger values of $g / t$ we find a more pronounced effect for such an incipient transition, the definite existence of which has been confirmed by our functional integral formulation of the BFM scenario [46]. In that study, the onset of the superconducting state for undoped systems $\left(n_{\mathrm{B}}=0.5\right)$ happens when $g / t$ is decreased to below $\approx 2$. For a hole doping corresponding to $n_{\mathrm{B}} \approx 0.4$, the superconducting state is stabilized for $g / t \leq 1$, which is quantitatively close to the result obtained for the finite sized ring-like structure. It is the relatively large intra-pair phase correlations (corresponding to the large value of $T^{*}$ ) which kill in this underdoped regime, $[3 / 8,4 / 8]$ for $n_{\mathrm{B}}$, the interpair phase correlations.

In Fig. 5, right part, we illustrate the variation of $T^{*}$ and $T_{\phi}$ as a function of the Feshbach resonance coupling strength $g / t$ for zero hole doping $\left(n_{\mathrm{B}}=0.5\right)$ - representing the undoped cuprates.

Without having had to assume any specific hole doping dependent $g / t$ in this BFM capturing the crystalline metastability of the cuprates, the results shown in Fig. 5 describe qualitatively correctly the physical features observed in the cuprates, which are inherent in their temperature - doping dependent phase diagram. It accounts for the hole doping induced phase transition between an insulating Bose glass and the superconducting state. Approaching the optimal hole doping rate, between $n_{\mathrm{B}}=0$ and $n_{\mathrm{B}}=1 / 8, T_{\mathrm{c}}$ becomes, as we can clearly see from the left panel of Fig. 5 to be determined by $T^{*}$. Upon further increasing the hole doping (decreasing $n_{\mathrm{B}}$ to below 1/8) the decreasing with increasing hole doping $T^{*}(x)$ forces $T_{\mathrm{c}}(x)$ to follow suit as expected for BCS superconductors controlled by the amplitude fluctuations encoded in the doping dependent $T^{*}(x)$.

\section{Quantum protection and the collective excitations of transient localized bound pairs}

The intricate physics of the HTSCs lies in their ability to form a superconducting state, starting from a system of localized transient spin-singlet electron-pairs with a fluctuating pairing amplitude which manifests itself in the opening of a pseudo-gap in the local singleparticle density of states of the electrons. In the process of fabricating such transient pairs, the initially itinerant electrons inside this pseudo-gap region lose any quasiparticle features. The spectral weight of electrons, having Fermi-liquid spectral properties, is zero in the energy regime marking this insulating pseudo-gap state. With decreasing the temperature, the broad rather structureless pseudo-gap spectral features of single particle states acquire upper and lower Bogoliubov branches [28]. Simultaneously, spatial phase coherence, linking transient pairs at neighbouring sites, generates collective Goldstone phase modes of a super-fluid condensate of transient real space pairs.

In order to highlight this intricate interplay between the spectral properties of the electrons and of the resonating transient bound pairs of them, we use a renormalization group procedure, which permits us to decouple the dynamics of the single-particle Fermionic entities from that of the two-particle Bosonic entities. Using Wegner's flow equation renormalization group technique [47], we transform $H_{\mathrm{BFM}}=H_{0}+H_{\mathrm{int}}$ :

$$
\begin{aligned}
& H_{0}=\sum_{\boldsymbol{k}, \sigma}\left(\varepsilon_{\boldsymbol{k} \sigma}-\mu\right) c_{\boldsymbol{k} \sigma}^{\dagger} c_{\boldsymbol{k} \sigma}+\sum_{\boldsymbol{q}}\left(E_{\boldsymbol{q}}-2 \mu\right) b_{\boldsymbol{q}}^{\dagger} b_{\boldsymbol{q}} \\
& H_{\mathrm{int}}=\frac{1}{\sqrt{N}} \sum_{\boldsymbol{k}, \boldsymbol{p}}\left(g_{\boldsymbol{k}, \boldsymbol{p}} b_{\boldsymbol{k}+\boldsymbol{p}} c_{\boldsymbol{k} \downarrow}^{\dagger} c_{\boldsymbol{p} \uparrow}^{\dagger}+g_{\boldsymbol{k}, \boldsymbol{p}}^{*} b_{\boldsymbol{k}+\boldsymbol{p}}^{\dagger} c_{\boldsymbol{p} \uparrow} c_{\boldsymbol{k} \downarrow}\right),
\end{aligned}
$$

in a sequence of infinitesimal steps, which describes the flow of the fermionic as well as bosonic dispersions $\varepsilon_{\boldsymbol{k} \sigma}(\ell)$ and $E_{\boldsymbol{q}}(\ell)$, with the exchange coupling constants $g_{k, p}(\ell)$, getting renormalized down to zero, at the fixed point where the flow-parameter $\ell$ reaches infinity. The flow equations which achieve that are given by $\partial_{\ell} H(\ell)=$ $[\eta(\ell), H(\ell)]$, with $\eta(\ell)=\left[H_{0}(\ell), H(\ell)\right]$, representing an anti-Hermitian generator and which has the quality that $\partial_{\ell} \operatorname{Tr}\left[H(\ell)-H_{0}(\ell)\right]^{2} \leq 0$. It is this which assures the total decoupling of bosonic and fermionic fields in the Hamiltonian. The residual interaction between them re-appears in form of the renormalized $\varepsilon_{\boldsymbol{k} \sigma}^{*}(\ell=\infty)$ and $E_{\boldsymbol{q}}^{*}(\ell=\infty)$, as well as in the renormalized fermion and boson operators, given by

$$
\begin{aligned}
& {\left[\begin{array}{c}
c_{-\boldsymbol{k},-\sigma}^{\dagger}(\ell) \\
c_{\boldsymbol{k}, \sigma}(\ell)
\end{array}\right]=u_{\boldsymbol{k}}^{\mathrm{F}}(\ell)\left[\begin{array}{c}
c_{-\boldsymbol{k},-\sigma}^{\dagger} \\
c_{\boldsymbol{k}, \sigma}
\end{array}\right]} \\
& \mp \frac{1}{\sqrt{N}} \sum_{\boldsymbol{q}} v_{\boldsymbol{k}, \boldsymbol{q}}^{\mathrm{F}}(\ell)\left[\begin{array}{c}
b_{\boldsymbol{q}}^{\dagger} c_{\boldsymbol{q}+\boldsymbol{k}, \sigma} \\
b_{\boldsymbol{q}} c_{\boldsymbol{q}-\boldsymbol{k},-\sigma}^{\dagger},
\end{array}\right], \\
& b_{\boldsymbol{q}}(\ell)=u_{\boldsymbol{q}}^{\mathrm{B}}(\ell) b_{\boldsymbol{q}}+\frac{1}{\sqrt{N}} \sum_{\boldsymbol{k}} v_{\boldsymbol{q}, \boldsymbol{k}}^{\mathrm{B}}(\ell) c_{\boldsymbol{k} \downarrow} c_{\boldsymbol{q}-\boldsymbol{k} \uparrow} .
\end{aligned}
$$

They determine the spectral properties of the system, as exemplified in Ref. [48]. $u_{k}^{\mathrm{F}, \mathrm{B}}(\ell)$ designate the spectral weights of the components of those fermionic, respectively bosonic excitations with well defined individual particle features. $v_{k, q}^{\mathrm{F}, \mathrm{B}}(\ell)$, on the contrary, designate the incoherent contributions of their spectral properties. The initial fermionic operators $c_{\boldsymbol{k}, \sigma}^{\dagger}$ describing 

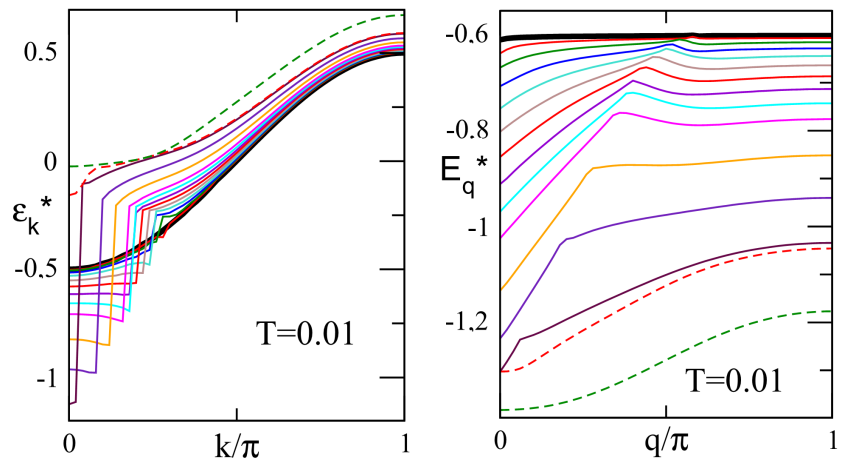

Fig. 6. The fermionic and bosonic fixed point dispersion $\varepsilon_{q}^{*}$ and $E_{q}^{*}$ at $T=0.01$ (after Ref. [49]) for $g=0.05$ (solid bold line), $g=$ $0.1,0.15,0.2,0.25,0.3,0.35,0.4,0.45,0.5,0.6,0.7,0.79$ (solid line) and $g=0.8,0.9$ (dashed line). The bare values are $\epsilon_{k}(\ell=0)=-2 t \cos k, E_{q}(\ell=0)=-0.6$ for $n_{\mathrm{F} \sigma}=n_{\mathrm{B}}=0.25$ implying $n_{\mathrm{tot}}=1$.

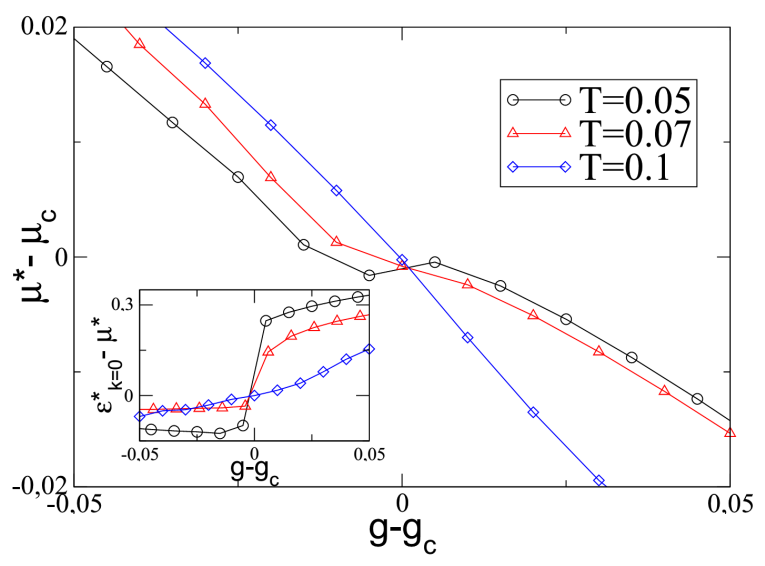

Fig. 7. Variation (after Ref. [49]) of the fixed point chemical potential $\mu^{*}$ with the Feshbach pair exchange coupling $g$ near its critical value $\mu_{\mathrm{c}}^{*}$ and $g_{\mathrm{c}}$ for a set of different temperatures. The changes from its monotonously decreasing behaviour into a nonmonotonous behaviour as $g$ varies from below to above a critical $g_{c}$ indicates the onset of a phase separation driven superconducting to non-superconducting state, similar to that of ${ }^{3} \mathrm{He}^{-}{ }^{4} \mathrm{He}$ mixtures.

itinerant electrons with a spectral weight $u_{k}^{\mathrm{F}}(\ell=0)=1$ get renormalized by the appearance of an extra term describing a hole travelling together with a bound electronpair $b_{\boldsymbol{q}}^{\dagger}$. As the temperature is lowered to below $T^{*}$, the spectral weight $u_{\boldsymbol{k}}^{\mathrm{F}}(\ell=\infty)$, describing the initially itinerant electrons, tends to zero and the pseudo-gap opens up in their density of states. The missing itinerant fermions have been eaten up in the process of constructing spectrally well defined bosonic bound pairs of them, with a dispersion, given by $E_{\boldsymbol{q}}^{*}(\ell=\infty)$. The electrons inside the pseudo-gap thereby lose any Fermi liquid features, as illustrated in Fig. 3. The localized bosonic excitations, characterized by their initial spectral weights
$u_{\boldsymbol{q}}^{\mathrm{B}}(\ell=0)=1, v_{\boldsymbol{q}}^{\mathrm{B}}(\ell=0)=0$, on the contrary, acquire a well defined collective linear in $\boldsymbol{q}$ Goldstone mode spectrum. It is triggered by the system's aspiration to condense into a super-fluid macroscopic coherent quantum state, controlled by what has been coined quantum protection [38, 39], as the temperature approaches $T_{\mathrm{c}}$. (see Fig. 6, right part). The transition from the superconducting state into the insulating pseudo-gap state is characterized by the chemical potential $\mu^{*}(\ell=\infty)$ moving out of the renormalized fermionic band $\varepsilon_{\boldsymbol{k}}^{*}$. It indicates that no fermionic excitations with well defined individual free particle features are left over, as illustrated in Fig. 6, left part. Simultaneously the transient bound pairs acquire a free particle behaviour with a $q^{2}$ spectrum. A more refined inspection of the insulatorsuperconductor phase transition [49] indicates that at low temperatures it is a first order phase transition, driven by a phase separation involving different relative concentrations of bound and unbound electron pairs. In order to determine whether the $q^{2}$ spectrum of $E_{\boldsymbol{q}}^{*}$ for transient phase uncorrelated bound electron pairs in the pseudogap state, actually are itinerant or diffusive modes, we will have to evaluate the auto-correlation function of the renormalized boson operators, $b_{\boldsymbol{q}}(l=\infty)$ in Eq. (12). This work is in progress.

\section{References}

[1] J. Ranninger, arXiv:1207.6911.

[2] H. Kamerlingh-Onnes, Acad. van Wettenschappen (Amsterdam) B 14, 113 (1911).

[3] J. Bardeen, L.N. Cooper, J.R. Schrieffer, Phys. Rev. 108, 1175 (1957).

[4] P. Morel, P.W. Anderson, Phys. Rev. 125, 1236 (1962).

[5] D. Pines, Ph. Nozieres, The Theory of Quantum Liquids, Benjamin, New York 1966.

[6] D.A. Kirzhnits, Usp. Fiz. Nauk 119, 357 (1976).

[7] O.V. Dolgov, D.A. Kirzhnits, E.G. Maksimov, Rev. Mod. Phys. 53, 81 (1981).

[8] V.L. Ginzburg, D.A. Kirszhnits, High Temperature Superconductivity, Consultant Bureau, New York 1982.

[9] B.T. Matthias, E. Corenzwit, A.S. Cooper, L.D. Longinotti, Proc. Natl. Acad. Sci. 68, 56 (1971).

[10] J.M. Vandenberg, B.T. Matthias, Science 198, 194 (1977).

[11] J.R. Galaver, Appl. Phys. Lett. 23, 480 (1973).

[12] S. Lakkis, C. Schlenker, B.K. Chakraverty, R. Buder, M. Marezzio, Phys. Rev. B 14, 1429 (1976).

[13] A.S. Alexandrov, J. Ranninger, Phys. Rev. B 23, 1796 (1981).

[14] R. Micnas, J. Ranninger, S. Robaszkiewicz, Rev. Mod. Phys. 62, 113 (1990).

[15] V.J. Emery, S.A. Kivelson, Nature (London) 374, 434 (1995).

[16] B.K. Chakraverty, J. Ranninger, D. Feinberg, Phys. Rev. Lett. 81, 433 (1998). 
[17] G. Bednorz, K.A. Müller, Z. Phys. B 64, 189 (1986).

[18] J.F. Schooly, W.R. Hosler, E. Ambler, J.H. Becker, M.L. Cohen, C.S. Koonce, Phys. Rev. Lett. 14, 305 (1965).

[19] R.J. Tainsh, C. Abdrikidis, Solid State Commun. 60, 517 (1986).

[20] D. Eagles, Phys. Rev. 186, 456 (1969).

[21] A.J. Leggett, J. Phys. C7 (France) 41, 19 (1980).

[22] Y.J. Uemura, G.M. Luke, B.J. Sternlieb, J.H. Brewer, J.F. Carolan, W.N. Hardy, R. Kadono, J.R. Kempton, R.F. Kiefl, S.R. Kreitzman, P. Mulhern, T.M. Riseman, D.L. Williams, B.X. Yang, S. Uchida, H. Takagi, J. Gopalakrishnan, A.W. Sleight, M.A. Subramanian, C.L. Chien, M.Z. Cieplak, Gang Xiao, V.Y. Lee, B.W. Statt, C.E. Stronach, W.J. Kossler, X.H. Yu, Phys. Rev. Lett. 62, 2317 (1989).

[23] P.W. Anderson, Science 235, 1196 (1987).

[24] E. Altman, A. Auerbach, Phys. Rev. B 65, 104508 (2002).

[25] J. Ranninger, S. Robaszkiewicz, Physica 135B, 468 (1985).

[26] J. Ranninger, R. Micnas, S. Robaszkiewicz, Ann. Phys. (France) 13, 455 (1989).

[27] J. Ranninger, J.-M. Robin, M. Eschrig, Phys. Rev. Lett. 74, 4027 (1995).

[28] T. Domanski, J. Ranninger, Phys. Rev. Lett. 91, 255301 (2003).

[29] P. Devillard, J. Ranninger, Phys. Rev. Lett. 84, 5200 (2000)

[30] C.J. Zhang, H. Oyanagi, Phys. Rev. B 79, 064521 (2009).

[31] D. Reznik, L. Pintschovius, M. Ito, S. Iikubo, M. Sato, H. Goka, M. Fujita, K. Yamada, G.D. Gu, J.M. Tranquada, Nature 440, 1170 (2006).

[32] Y. Kohsaka, C. Taylor, K. Fujita, A. Schmidt, C. Lupien, T. Hanaguri, M. Azuma, M. Takano, H. Eisaki, H. Takagi, S. Uchida, J.C. Davis, Science 315, 1380 (2007).
[33] Y. Kohsaka, Nature (London) 454, 1072 (2008).

[34] J. Lee, K. Fujita, A.R. Schmidt, Chung Koo Kim, H. Eisaki, S. Uchida, J.C. Davis, Science 325, 1099 (2009).

[35] J. Lee, K. Fujita, K. McElroy, J.A. Slezak, M. Wang, Y. Aiura, H. Bando, M. Ishikado, T. Masui, J.-X. Zhu, A.V. Balatsky, H. Eisaki, S. Uchida, J.C. Davis, $N a$ ture 442, 546 (2006).

[36] D. Rubio-Temprano, J. Mesot, S. Janssen, K. Conder, A. Furrer, H. Mutka, K.A. Müller, Phys. Rev. Lett. 84, 1990 (2000).

[37] P.S. Häfliger, A. Podlesnyak, K. Conder, A. Furrer, Eur. Phys. Lett. 73, 260 (2006).

[38] R.B. Laughlin, D. Pines, Proc. Natl. Acad. Sci. USA 97, 28 (2000).

[39] P.W. Anderson, Science 288, 480 (2000).

[40] L. Pauling, Nature of the Chemical Bond, Cornell University Press, Ithaca, New York 1960, p. 394.

[41] E.V.L. de Mello, J. Ranninger, Phys. Rev. B 55, 14872 (1997); Phys. Rev. B 58, 9098 (1998).

[42] H. Feshbach, Ann. Phys. (New York) 5, 357 (1958).

[43] T. Domanski, J. Ranninger, J.M. Robin, Solid State Commun. 105, 473 (1998).

[44] J. Ranninger, A. Romano, Phys. Rev. B 82, 054508 (2010).

[45] M. Cuoco, C. Noce, J. Ranninger, A. Romano, Phys. Rev. B 67, 224504 (2005).

[46] M. Cuoco, J. Ranninger, Phys. Rev. B 70, 104509 (2004).

[47] F. Wegner, Ann. Phys. (Leipzig) 3, 77 (1994).

[48] J. Ranninger, T. Domanski, Phys. Rev. B 81, 014514 (2010).

[49] T. Stauber, J. Ranninger, Phys. Rev. Lett. 99, 045301 (2007). 